\title{
Production of Kaon and $\Lambda$ in Nucleus-Nucleus Collisions at Ultrarelativistic Energy from a Blast-Wave Model
}

\author{
S. Zhang, ${ }^{1}$ Y. G. Ma, ${ }^{1,2}$ J. H. Chen, ${ }^{1}$ and C. Zhong ${ }^{1}$ \\ ${ }^{1}$ Shanghai Institute of Applied Physics, Chinese Academy of Sciences, Shanghai 201800, China \\ ${ }^{2}$ ShanghaiTech University, Shanghai 200031, China \\ Correspondence should be addressed to Y. G. Ma; ygma@sinap.ac.cn
}

Received 27 June 2014; Accepted 28 September 2014

Academic Editor: Bhartendu K. Singh

Copyright (c) 2015 S. Zhang et al. This is an open access article distributed under the Creative Commons Attribution License, which permits unrestricted use, distribution, and reproduction in any medium, provided the original work is properly cited. The publication of this article was funded by $\mathrm{SCOAP}^{3}$.

\begin{abstract}
The particle production of Kaon and $\Lambda$ is studied in nucleus-nucleus collisions at relativistic energy based on a chemical equilibrium blast-wave model. The transverse momentum spectra of Kaon and $\Lambda$ at the kinetic freeze-out stage from our model are in good agreement with the experimental results. The kinetic freeze-out parameters of temperature $\left(T_{\text {kin }}\right)$ and radial flow parameter $\rho_{0}$ are presented for the FOPI, RHIC, and LHC energies. And the resonance decay effect is also discussed. The systematic study for beam energy dependence of the strangeness particle production will help us to better understand the properties of the matter created in heavy-ion collisions at the kinetic freeze-out stage.
\end{abstract}

\section{Introduction}

Study on quark-gluon plasma (QGP) [1] by ultrarelativistic heavy-ion collisions [2-5] provides an opportunity to understand the production mechanism of strangeness hadrons and the phase-space evolution in the system created; in particular, the energy dependence is thought as one of important observables to study various aspects of the QCD phase diagram [6-8] in the beam energy scan program at RHIC [9]. The s-quarks in strangeness hadrons can be a good probe to investigate the properties of the high dense and temperature matter because there should be no net strangeness content in the colliding system [10-13]. The s-quarks are all created in the reaction. Kaon is produced associated with $\Lambda$-hyperon at FOPI energy and at higher energies, such as in the RHIC or LHC region, and it can be produced by another mechanism, the so-called pair production [2-5]. The transverse momentum $p_{T}$ distribution encodes rich physics information. It has been measured in $p+p, p+$ nucleus, or nucleus + nucleus collisions at different energy range by the FOPI [14] and KaoS [15] collaboration at $\sqrt{s_{N N}}=2.6 \mathrm{GeV}$, by the RHIC-STAR collaboration at $\sqrt{s_{N N}}=62 \mathrm{GeV}[16$, 17] and $200 \mathrm{GeV}[18,19]$, by the LHC-ALICE collaboration
[20-23] at $\sqrt{s_{N N}}=2.76$ and $7 \mathrm{TeV}$, and so forth. The resonance decay effect is also discussed in the above papers [16, 24]. Through fitting the measured transverse momentum $p_{T}$ distribution by a blast-wave model [25], one can investigate the properties of the collision system at kinetic freeze-out stage. The temperature $T_{\text {kin }}$ parameter and the radial flow at kinetic freeze-out stage can provide some information about the evolution of the expanding phase space. One can also study if the system has reached kinetic equilibrium stage when the hadron interactions were completed. The resonances decay will change the production rate and the $p_{T}$ distribution of Kaon and $\Lambda$. However, it is difficult to estimate the effect quantitatively in experiment. The blastwave model has been applied in experimental analysis $[16,26]$ to study the kinetic freeze-out properties. Retière and Lisa [27] have explored in detail an analytic parameterization of the freeze-out configuration and investigated the spectra, the collective flow, and the HBT correlation of the hadrons produced in head-on nuclear collisions at top RHIC energy. In addition, the DRAGON [28] and the THERMINATOR2 $[29,30]$ models have been developed to study the phasespace distribution of produced hadrons at freeze-out stage. In this paper, we carry out a detailed study on the beam 
energy dependence of Kaon and $\Lambda$ production based on a blast-wave model. The transverse momentum distributions of Kaon and $\Lambda$ are presented. The chemical and kinetic freeze-out parameters are consistent with the ones extracted from experimental data. The resonance decay effect is also investigated and an approximate estimation of the percent of Kaon and $\Lambda$ from resonance decay to total Kaon and $\Lambda$ is achieved.

\section{Blast-Wave Model with Thermal Equilibrium Mechanism}

As discussed above, within the framework of the blastwave model, the fireball created in high-energy heavy-ion collisions is assumed to be in local thermal equilibrium and expands at a four-component velocity $u_{\mu}$. The phase-space distribution of hadrons emitted from the expanding fireball can be expressed as a Wigner function [27-30]:

$$
\begin{aligned}
& S(x, p) d^{4} x \\
& =\frac{2 s+1}{(2 \pi)^{3}} m_{t} \cosh (y-\eta) \exp \left(-\frac{p^{\mu} u_{\mu}}{T_{\mathrm{kin}}}\right) \\
& \quad \Theta(1-\tilde{r}(r, \phi)) H(\eta) \delta\left(\tau-\tau_{0}\right) d \tau \tau d \eta r d r d \phi,
\end{aligned}
$$

where $s, y$, and $m_{t}$ are the spin, rapidity, and transverse mass of the hadron, respectively, and $p_{\mu}$ is the four-component momentum. Equation (1) is formulated in a Lorentz covariant way, $r$ and $\phi$ are the polar coordinates, and $\eta$ and $\tau$ are the pseudorapidity and the proper time, respectively. $\tilde{r}$ is defined as

$$
\tilde{r}=\sqrt{\frac{\left(x^{1}\right)^{2}}{R^{2}}+\frac{\left(x^{2}\right)^{2}}{R^{2}}}
$$

with $\left(x^{1}, x^{2}\right)$ standing for the coordinates in the transverse plane and $R$ being the average transverse radius. The kinetic freeze-out temperature $T_{\text {kin }}$ and the radial flow parameter $\rho_{0}$ are important in determining the transverse momentum spectrum. And the latter will affect the four-component velocity field. Since we are currently interested in the $p_{T}$ spectrum at midrapidity, the pseudorapidity distribution $H(\eta)$ is not important. The $p_{T}$ spectrum can then be written as

$$
\frac{d N}{2 \pi p_{T} d p_{T}}=\int S(x, p) d^{4} x .
$$

A thermal equilibrium model is employed to calculate the chemical components of hadrons. The particle density of species $i$ can be expressed as [28, 31-36]

$$
\begin{aligned}
& n_{i}\left(T_{\mathrm{ch}}, \mu_{B}, \mu_{S}\right) \\
& \quad=g_{i} \int \frac{d^{3} p}{(2 \pi)^{3}}\left[\exp \left(\frac{\sqrt{p^{2}+m_{i}^{2}}-\left(\mu_{B} B_{i}+\mu_{S} S_{i}\right)}{T_{\mathrm{ch}}}\right) \mp 1\right]^{-1} \\
& \quad=I\left(g_{i}, \frac{m_{i}}{T_{\mathrm{ch}}}\right) \sum_{n=1}( \pm 1)^{n+1} \exp \left(n \frac{\left(\mu_{B} B_{i}+\mu_{S} S_{i}\right)}{T_{\mathrm{ch}}}\right) \\
& I\left(g_{i}, \frac{m_{i}}{T_{\mathrm{ch}}}\right) \\
& \quad=g_{i} \int \frac{d^{3} p}{(2 \pi)^{3}}\left[\sum_{n=1}( \pm 1)^{n+1} \exp \left(-n \frac{\sqrt{p^{2}+m_{i}^{2}}}{T_{\mathrm{ch}}}\right)\right]
\end{aligned}
$$

with the upper (lower) sign being for bosons (fermions) and $g_{i}$ being the degeneracy factor. Assuming that the chemical equilibrium condition is satisfied, (4) essentially determines the fraction of particle species $i$. The particle yield can be described by adjusting parameters such as the chemical freeze-out temperature $T_{\mathrm{ch}}$, the baryon chemical potential $\mu_{B}$, the strangeness chemical potential $\mu_{S}$, and the system volume $V$. And then the fraction of particle species $i$ and its phasespace distribution can be calculated from (4) and (3).

\section{Results and Discussion}

The rapidity distributions of $K^{+}$, proton, and $\Lambda$ are presented in Figure 1. The hadron rapidity distribution is related to the density at reaction scope. The calculated results can describe the data from the FOPI [14] and KaoS collaborations [15] for $K^{+}$, proton, and $\Lambda$ in $\mathrm{Ni}+\mathrm{Ni}$ collisions at $\sqrt{s_{N N}} \simeq$ $2.6 \mathrm{GeV}$. The $\Lambda$ is mainly produced at midrapidity $(-0.5<$ $y<0.5)$ and is different from proton distribution at forward/backward rapidity. This results from the pronounced longitudinal expansion of proton and indicates large degree of transparency in $\mathrm{Ni}+\mathrm{Ni}$ system at the FOPI energies [14]. A similar calculation for $d N / d y$ from other groups can be found in [37] and our previous results for $\Lambda$ were calculated by a transport model [38].

The collective properties of the hot and dense matter created in ultrarelativistic heavy-ion collisions at freeze-out stage can be studied through transverse momentum $\left(p_{T}\right)$ distributions of identified particles. The radial flow

$$
\langle\beta\rangle=\frac{\int \operatorname{arctanh}\left(\rho_{0}(r / R)\right) r d r}{\int r d r}
$$

is related to the maximum flow rapidity

$$
\rho=\tilde{r}\left[\rho_{0}+\rho_{a} \cos (2 \phi)\right]
$$

The results are independent of $\rho_{a}$ since the $\phi$ dependence is averaged out after integration.

Transverse momentum $\left(p_{T}\right)$ spectrum is a basic observable in ultrarelativistic heavy-ion collisions $[39,40]$. By fitting 

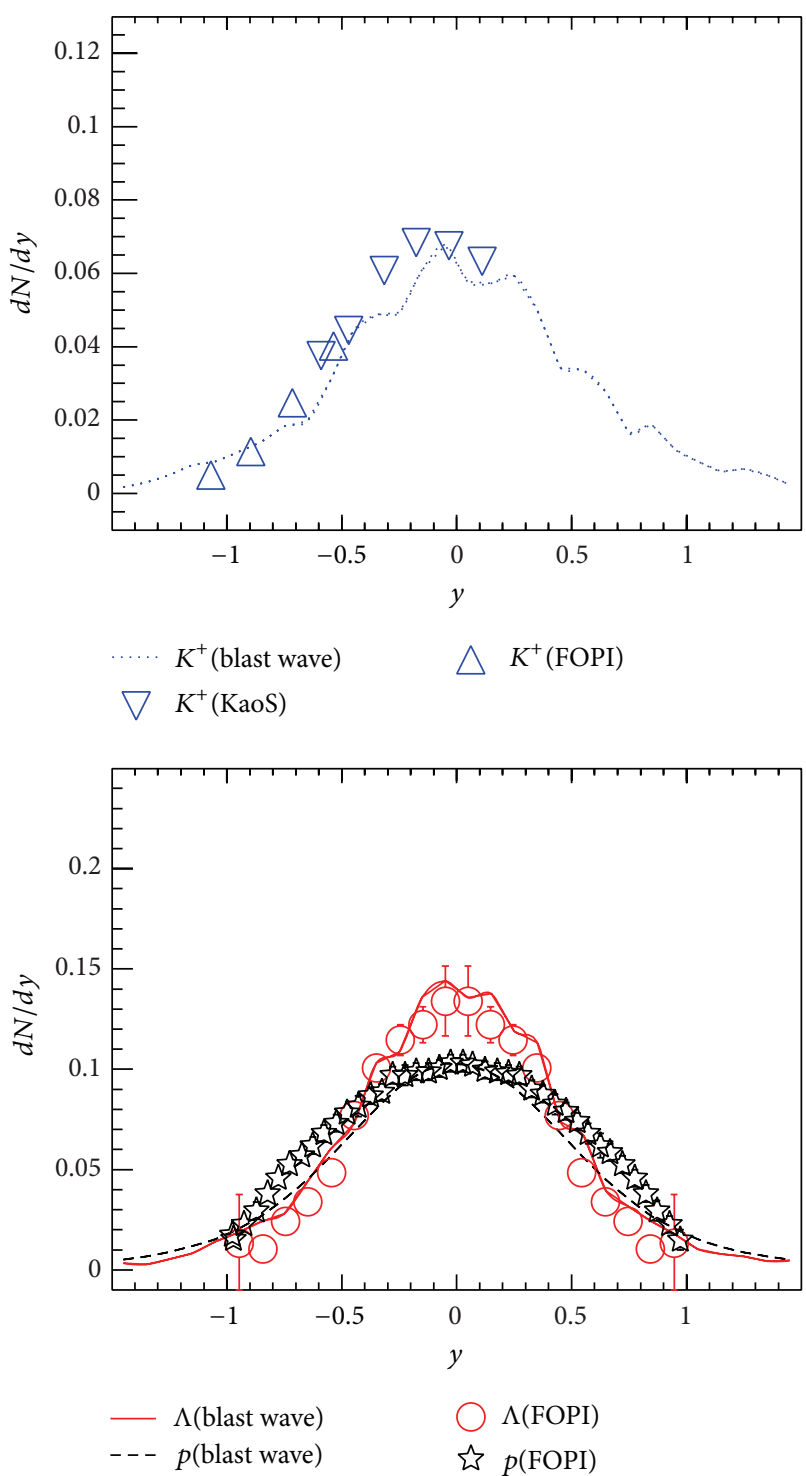

FIGURE 1: Rapidity distribution of $K^{+}, \Lambda$, and proton (normalized to $\Lambda$ yield) in $\mathrm{Ni}+\mathrm{Ni}$ central collisions at $\sqrt{s_{N N}}=2.6 \mathrm{GeV}$ (color online). Lines represent the model calculations with $T_{\text {kin }}=30 \mathrm{MeV}$, $\rho_{0}=0.6, T_{\mathrm{ch}}=49 \mathrm{MeV}$, and $\mu_{B}=815 \mathrm{MeV}$; symbols are data from the FOPI [14] and KaoS [15] experiments.

the transverse momentum spectra with thermal model, information of the system created in the collisions can be obtained, such as the kinetic freeze-out temperature and the radial flow. If the spectra are identified for different particles species, the ratio between the different particles will give the chemical freeze-out temperature and baryon (strangeness) chemical potentials. In this paper, a blast-wave model with chemical equilibrium model is employed to calculate the transverse momentum $\left(p_{T}\right)$ spectra of Kaon and $\Lambda$ in nucleus-nucleus collisions at ultrarelativistic energy $\sqrt{s_{N N}}$ from $2.6 \mathrm{GeV}$ to $2.76 \mathrm{TeV}$ corresponding to the FOPI, RHIC, and LHC energy range.

Figure 2 shows the transverse momentum distribution of $K^{0}$ and $\Lambda$ by the blast-wave model compared with the data

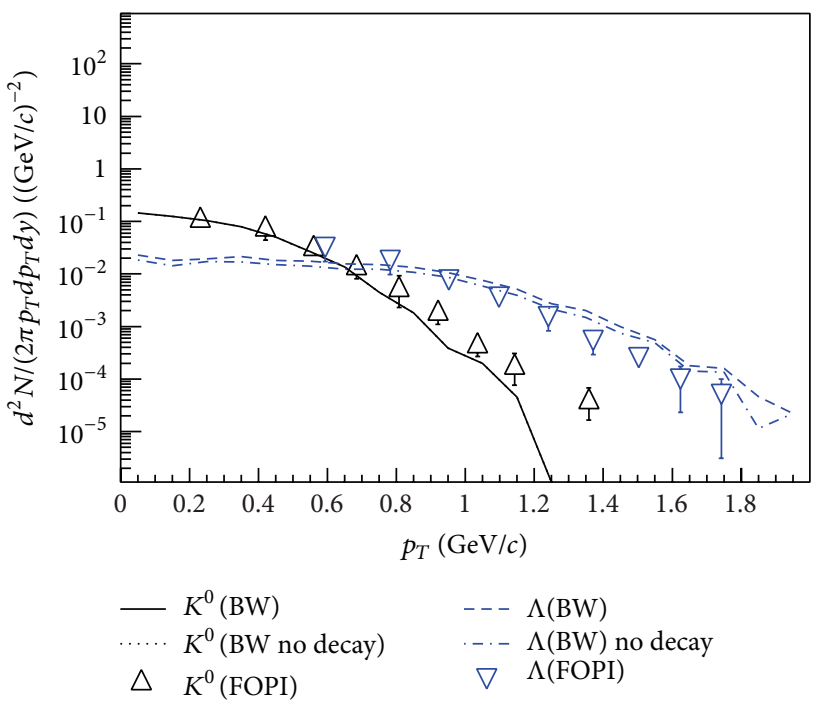

Figure 2: The transverse momentum $p_{T}$ distribution of $K^{0}$ and $\Lambda$ at midrapidity in $\mathrm{Ni}+\mathrm{Ni}$ central collisions at $\sqrt{s_{N N}} \simeq 2.6 \mathrm{GeV}$ (color online). Lines represent the model calculations with $T_{\text {kin }}=30 \mathrm{MeV}$, $\rho_{0}=0.6, T_{\mathrm{ch}}=49 \mathrm{MeV}$, and $\mu_{B}=815 \mathrm{MeV}$; symbols are data from the FOPI collaboration [14].

from FOPI collaboration [14] in Ni $+\mathrm{Ni}$ collisions at $\sqrt{s_{N N}} \simeq$ $2.6 \mathrm{GeV}$. Our results can describe the data well. The chemical temperature $T_{\mathrm{ch}}$ and the baryon chemical potential $\mu_{B}$ used in this calculation are similar to the ones applied in [37]. And the kinetic freeze-out parameters of temperature $T_{\text {kin }}$ and radial flow $\rho_{0}$ are chosen from experimental results with compilation in [16]. The production of Kaon and $\Lambda$ here is near the $\Lambda$ threshold; thus, it can be a reference in Lanzhou-Cooling Storage Ring (CSR) facility and High Intensity Accelerator Facility (HIAF) in the near future. Figures 3 and 4 present the transverse momentum distributions of $K^{+}$and $\Lambda$ at midrapidity in $\mathrm{Au}+\mathrm{Au}$ central collisions at $\sqrt{s_{N N}}=62.4 \mathrm{GeV}$ and $200 \mathrm{GeV}$, respectively. The chemical and kinetic freezeout parameters are extracted from data as in $[16,35,36]$. And for LHC energy the chemical freeze-out parameters and kinetic freeze-out temperatures were already discussed in our previous study [24] and Figure 5 presents the $p_{T}$ distribution of $K^{+}$and $\Lambda$ in $\mathrm{Pb}+\mathrm{Pb}$ central collisions at $\sqrt{s_{N N}} \simeq 2.76 \mathrm{TeV}$. Our calculated results are in good agreement with the $p_{T}$ distribution from experiment. From Figures 2, 3, 4, and 5, it can be found that the transverse momentum distributions of $\Lambda$ are harder than those of Kaon. The slope (effective temperature) of the momentum distribution is related to both the radial flow parameter $\langle\beta\rangle$ and the mass of particles $\left(m_{0}\right)$; that is, $T_{\text {eff }}=T_{\text {kin }}+(1 / 2) m_{0}\langle\beta\rangle^{2}[41-43]$. The radial flow will push the heavier particle to high transverse momentum; therefore, the stiffer spectrum emerges for $\Lambda$ in contrast with Kaon.

As discussed above the collective properties of the hot and dense matter created in ultrarelativistic heavy-ion collisions at freeze-out stage can be calculated through (5). Figure 6 shows the radial flow parameter $\langle\beta\rangle$ as a function of centre of mass energy $\sqrt{s_{N N}}$. It shows an increasing trend 


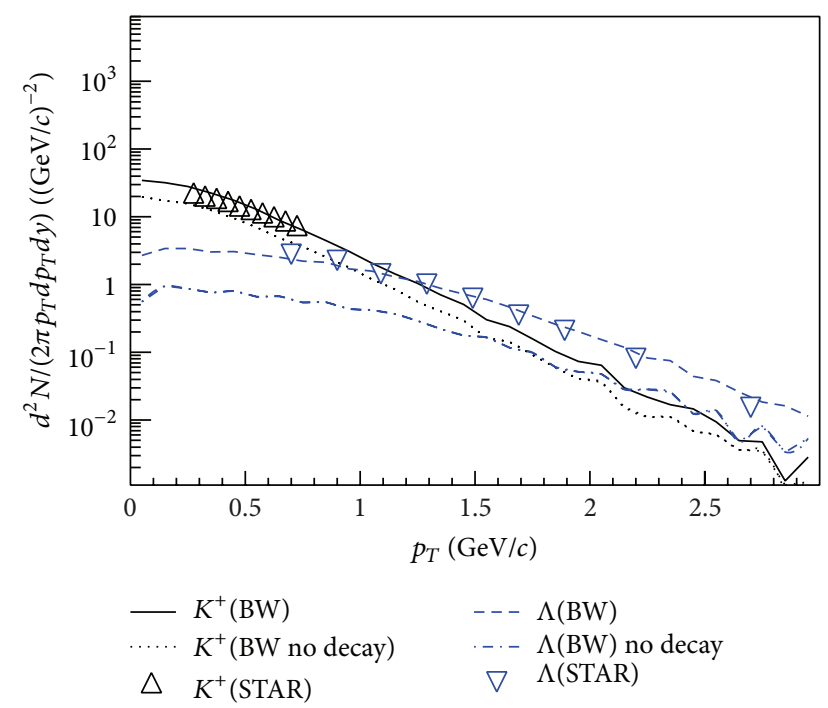

FIgURE 3: The transverse momentum $p_{T}$ distribution of $K^{+}$and $\Lambda$ at midrapidity in $\mathrm{Au}+\mathrm{Au}$ central collisions at $\sqrt{{s_{N N}}}=62.4 \mathrm{GeV}$ (color online). Lines represent the model calculations with $T_{\text {kin }}=105 \mathrm{MeV}$, $\rho_{0}=0.7, T_{\mathrm{ch}}=156 \mathrm{MeV}$, and $\mu_{B}=70 \mathrm{MeV}$; symbols are data from the RHIC-STAR collaboration $[16,17]$.

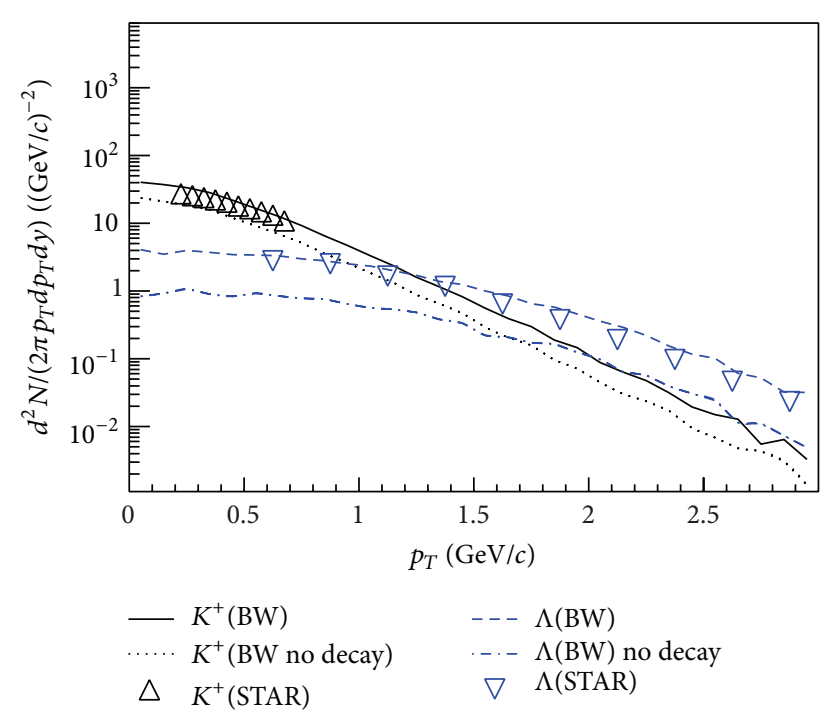

FIgURE 4: The transverse momentum $p_{T}$ distribution of $K^{+}$and $\Lambda$ at midrapidity in $\mathrm{Au}+\mathrm{Au}$ central collisions at $\sqrt{s_{N N}}=200 \mathrm{GeV}$ (color online). Lines represent the model calculations with $T_{\text {kin }}=91 \mathrm{MeV}$, $\rho_{0}=0.8, T_{\mathrm{ch}}=156 \mathrm{MeV}$, and $\mu_{B}=20 \mathrm{MeV}$; symbols are data from the RHIC-STAR collaboration $[18,19]$.

of the radial flow with the increasing of centre of mass energy $\sqrt{s_{N N}}$. Namely, the expanding velocity of the collision system in radial direction is larger at higher energy. From Figure 6, the radial flow used in this calculation is consistent with those extracted from the experimental results [16]. It implies that these parameters are also valid for strangeness hadrons.

The resonance production rate is high in nucleus-nucleus collisions at relativistic energy. The hadrons decayed from

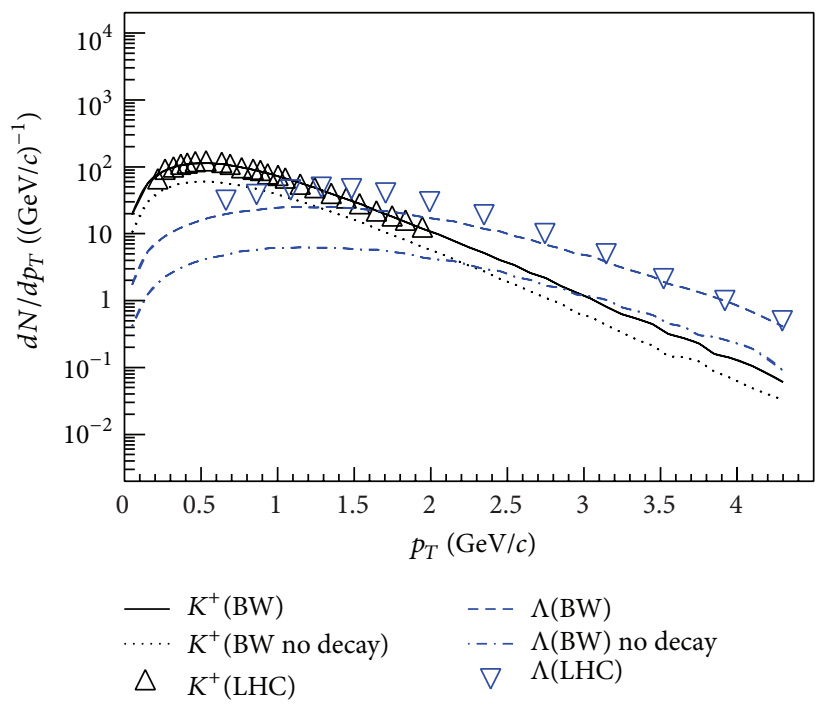

Figure 5: The transverse momentum $p_{T}$ distribution of $K^{+}$and $\Lambda$ at midrapidity in $\mathrm{Pb}+\mathrm{Pb}$ central collisions at $\sqrt{s_{N N}}=2.76 \mathrm{TeV}$ (color online). Lines represent the model calculations with $T_{\text {kin }}=99 \mathrm{MeV}$, $\rho_{0}=1, T_{\mathrm{ch}}=160 \mathrm{MeV}$, and $\mu_{B}=10 \mathrm{MeV}$; experimental data are taken from $[20,21]$.

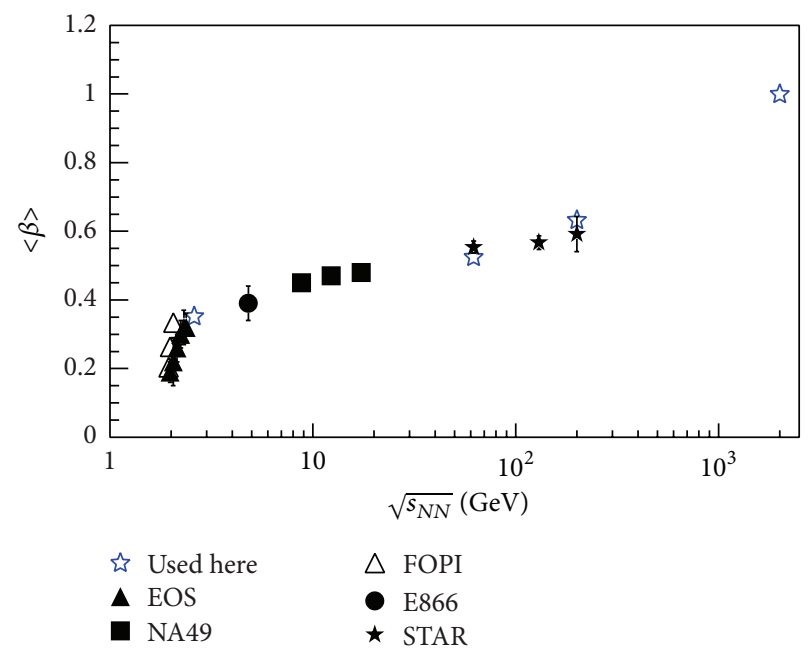

FIGURE 6: The radial flow parameters as a function of centre of mass energy $\sqrt{s_{N N}}$; data are taken from the RHIC-STAR collaboration [18, 19] (color online).

resonances will pollute the $p_{T}$ distribution of hadrons produced in the collision [16]. However, it is difficult to measure the effect precisely in experiment due to the wide species of resonance. This can be done in model calculation, though. In our previous work [24], this effect for $\pi, K$, and $p$ is discussed in detail in the LHC energy range. We show the results for strangeness hadron in different energy from Figures 2, 3,4 , and 5 in this paper. In this calculation, Kaon's and $\Lambda$ 's resonances are all included. The Kaons (decayed from resonances) are mainly from $K^{*}(892), \phi(1020)$, and other heavy resonances whose masses are heavier than that of 2 Kaons, and $\Lambda$ 's (decayed from resonance) are mainly from $\Sigma$ 's 
TABLE 1: Percent of $K^{+}$and $\Lambda$ from resonance decay in total $K^{+}$and $\Lambda$ versus centre of mass energy $\sqrt{s_{N N}}$.

\begin{tabular}{lcccc}
\hline$\sqrt{s_{N N}}(\mathrm{GeV})$ & 2.6 & 62 & 200 & 2000 \\
\hline$K^{+}$(decayed)/K (total) [\%] & 0.2 & 44.4 & 44.6 & 46.9 \\
$\Lambda$ (decayed) $/ \Lambda$ (total) [\%] & 19.5 & 73.8 & 74.1 & 74.9 \\
\hline
\end{tabular}

and $\Xi$ 's resonances. From (4) and the mass of those particles mentioned above, one can estimate that the production rate from resonance decay of $\Lambda$ 's will be more than that of Kaons. And Table 1 summarises our estimation for this effect. The percent of $K^{+}(\Lambda)$ from resonance decay to total $K^{+}(\Lambda)$ is increased with the increasing of centre of mass energy $\sqrt{s_{N N}}$. The percentage for $K^{+}$is higher than that for $\Lambda$. And the effect from resonance decay to $K^{+}$and $\Lambda$ is more significant at RHIC and LHC energy than the ones at low beam energy.

\section{Summary}

The blast-wave model with a chemical equilibrium model is employed to calculate the production of Kaon and $\Lambda$ in nucleus-nucleus collisions at relativistic energy. The baryon chemical potential $\mu_{B}$ and chemical freeze-out temperature $T_{\mathrm{ch}}$ are consistent with those extracted from experiments [16] and used in other model calculations [37]. The kinetic freeze-out properties are also discussed such as kinetic freezeout temperature $T_{\text {kin }}$ and radial flow parameter $\rho_{0}$. These kinetic freeze-out parameters are in good agreement with the ones from experimental results and are found to be valid for strangeness hadrons (Kaon and $\Lambda$ ). And the resonance decay effect on strangeness production is also studied in detail in this paper. In summary, the beam energy dependence of Kaon and $\Lambda$ production can be a good probe to study the properties of the dense matter created in ultrarelativistic heavy-ion collisions. Our results shed light on the beam energy scan at RHIC and the academic activity related to strangeness production at Lanzhou-CSR facility.

\section{Conflict of Interests}

The authors declare that there is no conflict of interests regarding the publication of this paper.

\section{Acknowledgments}

This work was supported in part by the Major State Basic Research Development Program in China under Contract no. 2014CB845400, the National Natural Science Foundation of China under Contract nos. 11421505, 11035009, 11220101005, $11105207,11275250,11322547$, and U1232206, and the Knowledge Innovation Project of the Chinese Academy of Sciences under Grant no. KJCX2-EW-N01.

\section{References}

[1] F. Karsch, "Lattice results on QCD thermodynamics," Nuclear Physics A, vol. 698, no. 1-4, pp. 199-208, 2002.
[2] I. Arsene, I. G. Bearden, D. Beavis et al., "Quark-gluon plasma and color glass condensate at RHIC? The perspective from the BRAHMS experiment," Nuclear Physics A, vol. 757, no. 1-2, pp. 1-27, 2005.

[3] B. B. Back, M. D. Baker, M. Ballintijn et al., "The PHOBOS perspective on discoveries at RHIC," Nuclear Physics A, vol. 757, no. 1-2, pp. 28-101, 2005.

[4] J. Adames, M. M. Aggarwal, Z. Ahammed et al., "Experimental and theoretical challenges in the search for the quark-gluon plasma: the STAR Collaboration's critical assessment of the evidence from RHIC collisions," Nuclear Physics A, vol. 757, no. 1-2, pp. 102-183, 2005.

[5] K. Adcox, S. S. Adler, S. Afanasiev et al., "Formation of dense partonic matter in relativistic nucleus-nucleus collisions at RHIC: experimental evaluation by the PHENIX Collaboration," Nuclear Physics A, vol. 757, no. 1-2, pp. 184-283, 2005.

[6] J. Tian, J. H. Chen, Y. G. Ma et al., "Breaking of the numberof-constituent-quark scaling for identified-particle elliptic flow as a signal of phase change in low-energy data taken at the BNL Relativistic Heavy Ion Collider (RHIC)," Physical Review C: Nuclear Physics, vol. 79, no. 6, Article ID 067901, 2009.

[7] F. M. Liu, "Explore QCD phase transition with thermal photons," Nuclear Science and Techniques, vol. 24, Article ID 050524, 2013.

[8] C. M. Ko, L. W. Chen, V. Greco et al., "Mean-field effects on matter and antimatter elliptic flow," Nuclear Science and Techniques, vol. 24, no. 5, Article ID 050525, 2013.

[9] B. Mohanty, "STAR experiment results from the beam energy scan program at the RHIC," Journal of Physics G: Nuclear and Particle Physics, vol. 38, no. 12, Article ID 124023, 2011.

[10] B. I. Abelev, "Partonic flow and $\phi$-Meson production in $\mathrm{Au}+\mathrm{Au}$ collisions at $\sqrt{s_{N N}}=200 \mathrm{GeV}$," Physical Review Letters, vol. 99, no. 11, Article ID 112301, 6 pages, 2007.

[11] Y. G. Ma, “ $\phi$-meson production and partonic collectivity at RHIC," Journal of Physics G: Nuclear and Particle Physics, vol. 32, no. 12, article S373, 2006.

[12] S. Zhang, J. H. Chen, H. Crawford, D. Keane, Y. G. Ma, and Z. B. Xu, "Searching for onset of deconfinement via hypernuclei and baryon-strangeness correlations," Physics Letters B: Nuclear, Elementary Particle and High-Energy Physics, vol. 684, no. 4-5, pp. 224-227, 2010.

[13] J. H. Chen, F. Jin, D. Gangadharan, X. Z. Cai, H. Z. Huang, and Y. G. Ma, "Parton distributions at hadronization from bulk dense matter produced in $\mathrm{Au}+\mathrm{Au}$ collisions at $\sqrt{s_{N N}}=200 \mathrm{GeV}$," Physical Review C, vol. 78, no. 3, Article ID 034907, 2008.

[14] M. Merschmeyer, X. Lopez, N. Bastid et al., " $K$ and $\Lambda$ production in Ni+Ni collisions near threshold," Physical Review C, vol. 76, Article ID 024906, 2007.

[15] A. Förster, F. Uhlig, I. Böttcher et al., "Production of $K^{+}$and of $K^{-}$mesons in heavy-ion collisions from $0.6 \mathrm{~A}$ to $2.0 \mathrm{~A} \mathrm{GeV}$ incident energy," Physical Review C: Nuclear Physics, vol. 75, no. 2, Article ID 024906, 2007.

[16] B. I. Abelev, M. M. Aggarwal, Z. Ahammed et al., "Systematic measurements of identified particle spectra in $p p, d+\mathrm{Au}$, and 
$\mathrm{Au}+\mathrm{Au}$ collisions at the STAR detector," Physical Review C, vol. 79, Article ID 034909, 2009.

[17] M. M. Aggarwal, Z. Ahammed, (STAR Collaboration) et al., "Strange and multistrange particle production in $\mathrm{Au}+\mathrm{Au}$ collisions at $\sqrt{s_{N N}}=62.4 \mathrm{GeV}$," Physical Review $C$, vol. 83, Article ID 024901, 2011.

[18] J. Adams, C. Adler, M. M. Aggarwal et al., "Identified particle distributions in $p p$ and $\mathrm{Au}+\mathrm{Au}$ collisions $\sqrt{s_{N N}}=200 \mathrm{GeV}$," Physical Review Letters, vol. 92, Article ID 112301, 2004.

[19] G. Agakishiev, M. M. Aggarwal, Z. Ahammed et al., "Strangeness enhancement in $\mathrm{Cu}-\mathrm{Cu}$ and $\mathrm{Au}-\mathrm{Au}$ collisions at $\sqrt{s_{N N}}=200 \mathrm{GeV}$," Physical Review Letters, vol. 108, no. 7, Article ID 072301, 6 pages, 2012.

[20] B. Müller, J. Schukraft, and B. Wysłouch, "First results from $\mathrm{Pb}+\mathrm{Pb}$ collisions at the LHC," Annual Review of Nuclear and Particle Science, vol. 62, pp. 361-386, 2012.

[21] D. D. Chinellato, "Strange and multi-strange particle production in ALICE," Journal of Physics: Conference Series, vol. 446, no. 1, Article ID 012055, 2013.

[22] K. Aamodt, N. Abel, U. Abeysekara et al., "Charged-particle multiplicity measurement in proton-proton collisions at $\sqrt{s}=7$ TeV with ALICE at LHC," The European Physics Journal C, vol. 68, no. 3-4, pp. 345-354, 2010.

[23] H. M. Wang, Z. Y. Hou, and X. J. Sun, "Hadron multiplicities in $\mathrm{p}+\mathrm{p}$ and $\mathrm{p}+\mathrm{Pb}$ collisions at the LHC," Nuclear Science and Techniques, vol. 25, Article ID 040502, 2014.

[24] S. Zhang, L. X. Han, Y. G. Ma et al., "Production and ratio of $\pi$, $K$, $p$, and $\Lambda$ in $\mathrm{Pb}+\mathrm{Pb}$ collisions at $\sqrt{s_{N N}}=2.76 \mathrm{TeV}$," Physical Review C, vol. 89, no. 3, Article ID 034918, 8 pages, 2014.

[25] E. Schnedermann, J. Sollfrank, and U. Heinz, "Thermal phenomenology of hadrons from 200A GeV S+S collisions," Physical Review C, vol. 48, no. 5, pp. 2462-2475, 1993.

[26] B. Abelev et al., "Centrality dependence of $\pi, K$, and $p$ production in $\mathrm{Pb}-\mathrm{Pb}$ collisions at $\sqrt{s_{N N}}=2.76 \mathrm{TeV}$," Physical Review C, vol. 88, Article ID 044910, 2013.

[27] F. Retière and M. A. Lisa, "Observable implications of geometrical and dynamical aspects of freeze-out in heavy ion collisions," Physical Review C, vol. 70, no. 4, Article ID 044907, 2004.

[28] B. Tomášik, "DRAGON: Monte Carlo generator of particle production from a fragmented fireball in ultrarelativistic nuclear collisions," Computer Physics Communications, vol. 180, no. 9, pp. 1642-1653, 2009.

[29] M. Chojnacki, A. Kisiel, W. Florkowski, and W. Broniowski, "THERMINATOR 2: THERMal heavy IoN generATOR 2," Computer Physics Communications, vol. 183, no. 3, pp. 746-773, 2012.

[30] A. Kisiel, T. Tałuć, W. Broniowski, and W. Florkowski, "THERMINATOR: THERMal heavy-IoN generATOR," Computer Physics Communications, vol. 174, no. 8, pp. 669-687, 2006.

[31] P. Braun-Munzinger, J. Stachel, J. P. Wessels, and N. Xu, “Thermal equilibration and expansion in nucleus-nucleus collisions at the AGS," Physics Letters B: Nuclear, Elementary Particle and High-Energy Physics, vol. 344, no. 1-4, pp. 43-48, 1995.

[32] P. Braun-Munzinger, J. Stachel, J. P. Wessels, and N. Xu, "Thermal and hadrochemical equilibration in nucleus-nucleus collisions at the SPS," Physics Letters, Section B: Nuclear, Elementary Particle and High-Energy Physics, vol. 365, no. 1-4, pp. 1-6, 1996.

[33] P. Braun-Munzinger, I. Heppe, and J. Stachel, "Chemical equilibration in $\mathrm{Pb}+\mathrm{Pb}$ collisions at the SPS," Physics Letters B: Nuclear, Elementary Particle and High-Energy Physics, vol. 465, no. 1-4, pp. 15-20, 1999.
[34] N. Xu and M. Kaneta, "Hadron freeze-out conditions in high energy nuclear collisions," Nuclear Physics A, vol. 698, no. 1-4, pp. 306-313, 2002.

[35] P. Braun-Munzinger, K. Redlich, and J. Stachel, "Particle production in heavy ion collisions," http://arxiv.org/abs/nucl-th/ 0304013.

[36] A. Andronic, P. Braun-Munzinger, and J. Stachel, "Thermal hadron production in relativistic nuclear collisions: The hadron mass spectrum, the horn, and the QCD phase transition," Physics Letters B, vol. 673, no. 2, pp. 142-145, 2009.

[37] A. Andronic, P. Braun-Munzinger, and J. Stachel, "Hadron production in central nucleus-nucleus collisions at chemical freeze-out," Nuclear Physics A, vol. 772, no. 3-4, pp. 167-199, 2006.

[38] S. Zhang, J.-H. Chen, Y.-G. Ma et al., "Hypertriton and light nuclei production at $\Lambda$-production subthreshold energy in heavy-ion collisions," Chinese Physics C, vol. 35, no. 8, pp. 741746, 2011.

[39] X.-N. Wang and M. Gyulassy, "Hijing: a Monte Carlo model for multiple jet production in pp, pA, and AA collisions," Physical Review D, vol. 44, no. 11, pp. 3501-3516, 1991.

[40] F.-H. Liu, Y.-Q. Gao, T. Tian, and B.-C. Li, “Transverse momentum and pseudorapidity distributions of charged particles and spatial shapes of interacting events in $\mathrm{Pb}-\mathrm{Pb}$ Collisions at 2.76TeV," Advances in High Energy Physics, vol. 2014, Article ID 725739, 14 pages, 2014.

[41] R. Scheibl and U. Heinz, "Coalescence and flow in ultrarelativistic heavy ion collisions," Physical Review C, vol. 59, no. 3, pp. 1585-1602, 1999.

[42] U. Heinz, "The little bang: searching for quark-gluon matter in relativistic heavy-ion collisions," Nuclear Physics A, vol. 685, no. $1-4,2001$.

[43] N. Xu and M. Kaneta, "Hadon freeze-out conditions in high energy nuclear collisions," Nuclear Physics A, vol. 698, p. 360c, 2002. 

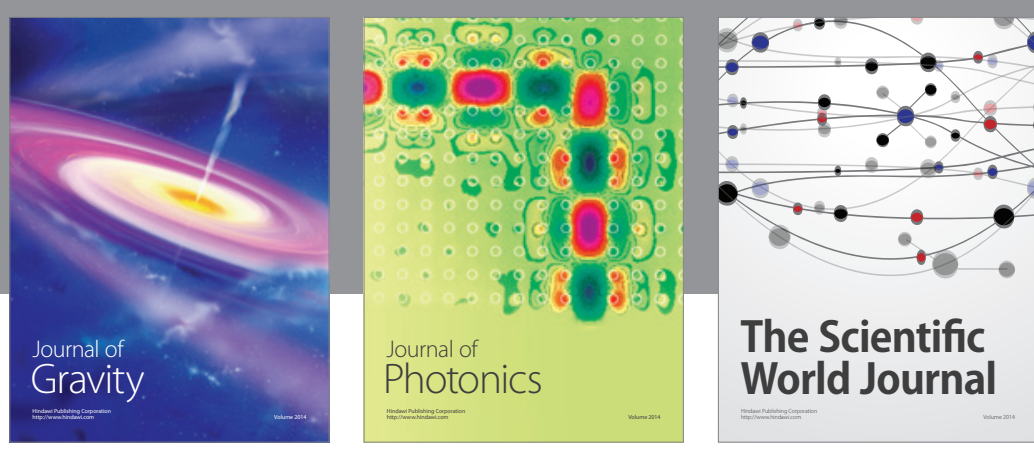

The Scientific World Journal
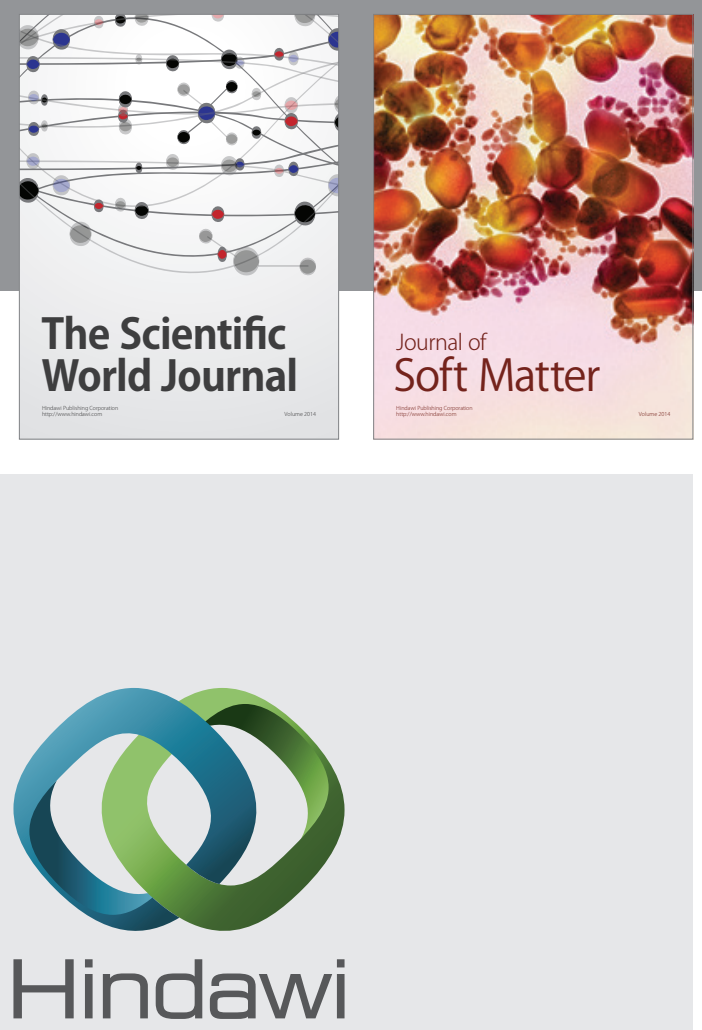

Submit your manuscripts at

http://www.hindawi.com

nternational Journal of

Statistical Mechanics
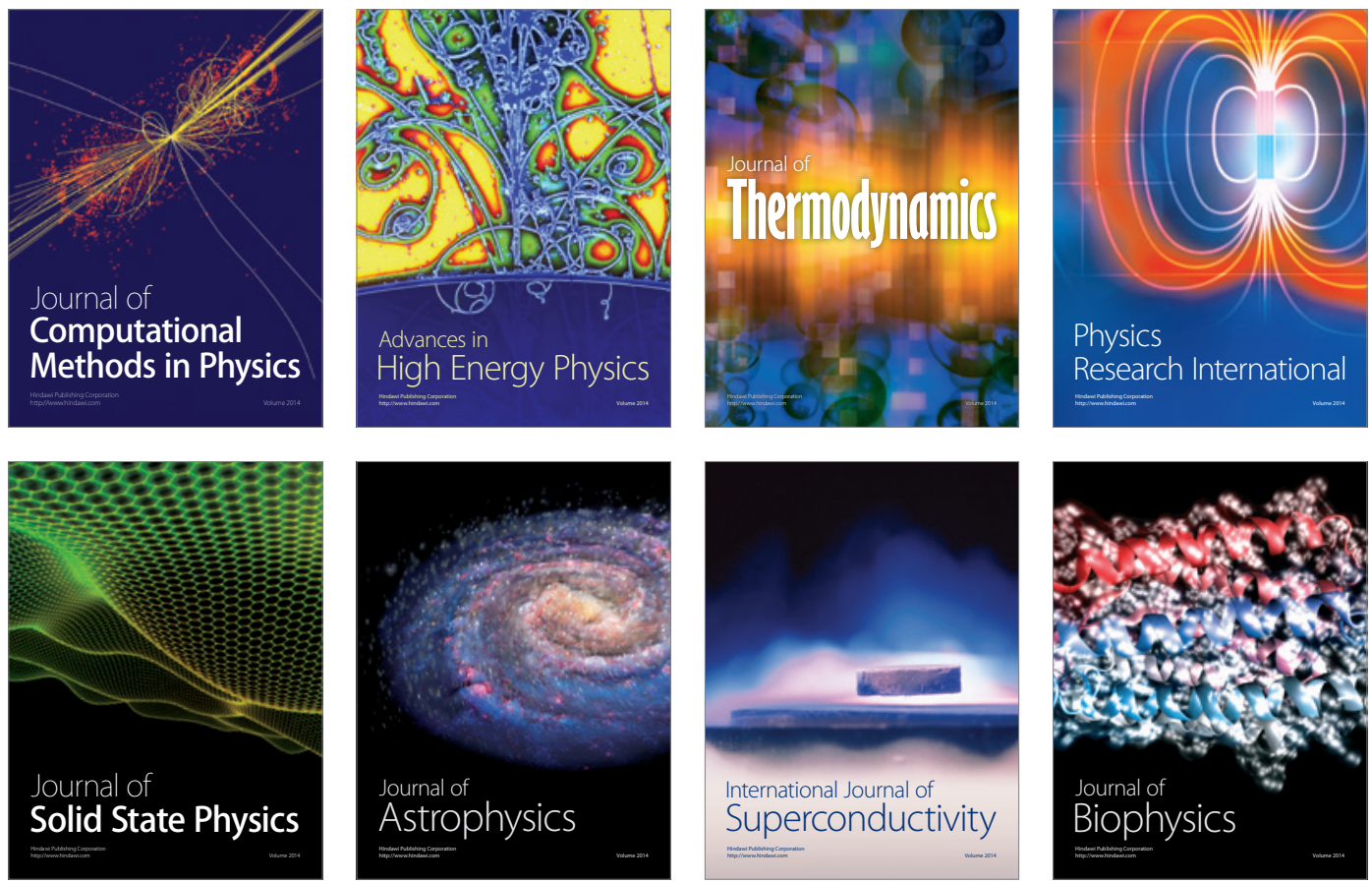
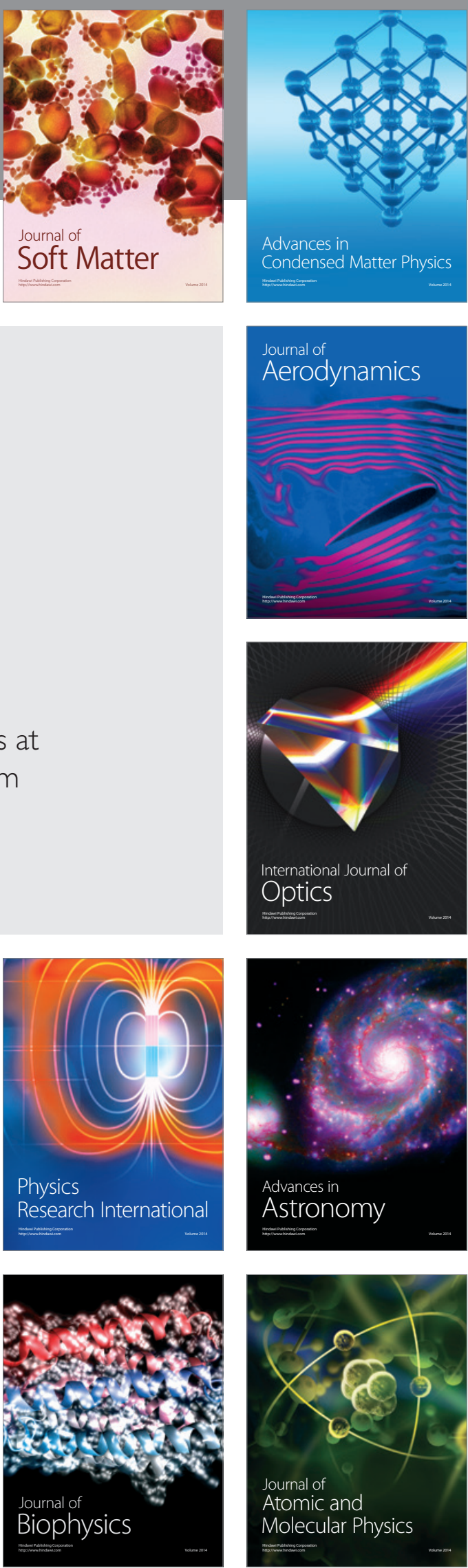